\title{
Some Thoughts upon Long-Range Interaction and Entangled States
}

\author{
Bo Lehnert \\ Alfvén Laboratory, Royal Institute of Technology, Stockholm, Sweden \\ Email: bo.lehnert@ee.kth.se
}

How to cite this paper: Lehnert, B. (2018) Some Thoughts upon Long-Range Interaction and Entangled States. Journal of Electromagnetic Analysis and Applications, 10, 193-196.

https://doi.org/10.4236/jemaa.2018.1012015

Received: November 15, 2018

Accepted: December 24, 2018

Published: December 27, 2018

Copyright $\odot 2018$ by author and Scientific Research Publishing Inc. This work is licensed under the Creative Commons Attribution International License (CC BY 4.0).

http://creativecommons.org/licenses/by/4.0/

\begin{abstract}
The problems of long-range interaction and associated questions on entangled states are reconsidered in terms of a recently developed revised quantum electrodynamic theory by the author, as being applied to subatomic systems. There are indications that the theories of relativity and quantum mechanics do not necessarily have to be in conflict. But more investigations are required for a full understanding to be obtained on these problems.
\end{abstract}

\section{Keywords}

Revised Quantum Electrodynamics, Standard Model and Beyond, Zero Point Energy, Long-Range Interaction, Entangled States

\section{Introduction}

One of the remaining problems of fundamental theoretical physics is the apparent contradiction between the limited characteristic velocity of special relativity and the instantaneous behaviour of entangled states through long-range interaction in quantum mechanics. In the present investigation, an attempt is made to dissolve this contradiction, in terms of an earlier revised quantum electrodynamic theory (RQED) by the author [1] [2], being applied to subatomic systems, such as the interior of elementary particles. Thereby, the vacuum does not become a state of empty space, but is populated by Zero Point Energy modes which also can become electrically polarized to generate electric charges and currents.

In Section 2, a summary is given on resulting modes of the revised theory. This is followed in Section 3 by considerations on long-range interaction between spatially limited particle configurations which may take place in terms of these modes. 


\section{Modes of the Revised Theory}

Depending on the concepts $\operatorname{div} \mathbf{E}$ and curl $\mathbf{E}$ of the electric field strength $\mathbf{E}$, there are three types of modes in RQED theory [1] [2].

\subsection{The EM Mode}

When $\operatorname{div} \mathbf{E}=0$ and curl $\mathbf{E} \neq 0$ a conventional electromagnetic EM mode arises which satisfies special relativity. It concerns a vacuum state of empty space, with no sources of electric charges and currents. This mode is transverse and does not possess any angular momentum (spin), as shown in Chapter 9 of Reference [1]. There is no steady particle state.

\subsection{The EMS Mode}

When $\operatorname{div} \mathbf{E} \neq 0$ due to arguments based on Zero Point Energy in the vacuum state, and when curl $\mathbf{E} \neq 0$ simultaneously, there is an EMS mode. It is of nontransverse and electromagnetic space-charge character, and also becomes consistent with special relativity. In axisymmetric geometry the wave velocity has two components, one along the direction of propagation and one circulating in the transverse direction. The latter gives rise to a nonzero spin, also in a steady state, as determined by the electrically polarized Zero Point Energy populating the vacuum state. Both as a wave and as a steady particle model the EMS mode includes a longitudinal electric field component, i.e. along the axis of symmetry.

The equations of the relativistic EM and EMS modes include the dielectric constant $\varepsilon_{0}$, the magnetic permeability $\mu_{0}$, and the limiting velocity $c=\left(1 / \varepsilon_{0} \mu_{0}\right)^{1 / 2}$. The two velocity components of the EMS mode are then defined by a velocity vector $\mathbf{C}=c(0, \cos \theta, \sin \theta)$ in a cylindrical frame. For the electric field we have

$$
\left(\frac{\partial^{2}}{\partial t^{2}}-c^{2} \nabla^{2}\right) \mathbf{E}+\left(c^{2} \nabla+\boldsymbol{C} \frac{\partial}{\partial t}\right)(\operatorname{div} \mathbf{E})=0
$$

where the first term is related to $\operatorname{curl} \mathbf{E}$.

\subsection{The S Mode}

When $\operatorname{div} \mathbf{E} \neq 0$ and $\operatorname{div} \mathbf{E}=0$ there remains an electric longitudinal space-charge mode. This mode is produced by the Zero Point Energy in vacuo, and has no relation to the theory of relativity. In the field equations all terms with the magnetic field $\mathbf{B}$ and the constant $\mu_{0}$ vanish. Only an electric field $\mathbf{E}=-\nabla \phi$ remains. Equation (1) then reduces to

$$
\left(c^{2} \nabla+\boldsymbol{C} \frac{\partial}{\partial t}\right)(\operatorname{div} \mathbf{E})=0
$$

with $\mathbf{C}=\left(C_{r}, 0, C_{z}\right)$ in a cylindrical frame. For this mode the components $C_{r}$ and $C_{z}$ can be arbitrarily chosen. This has nothing to do with special relativity and the velocity $c$. In other words, the EM and EMS modes can remain 
relativistic, independently of the $S$ mode.

With a normal mode representation where $\partial / \partial t=i \omega$ and $\partial / \partial z=i k$ Equation (2) yields

$$
\left(-\frac{\partial^{2} \phi}{\partial r^{2}}-\frac{1}{r} \frac{\partial \phi}{\partial r}+k^{2} \phi\right)\left(C_{r}, C_{z}\right)=\omega\left(i \frac{\partial \phi}{\partial r},-k \phi\right)
$$

Here, it is seen that a given potential $\phi$ cannot satisfy both these equations simultaneously. An S mode does therefore not exist in this general case. But for a flat geometry where $\phi$ is independent of $r$, a solution may still exist, as given by $C_{r}=0$ with the relation

$$
k C_{z}=-\omega
$$

We are then free to choose $C_{z}<0$ and $\left|C_{z}\right| / c \gg 1$ in a superluminal range. For a finite frequency $\omega$, the wave number $k$ then becomes very small and the corresponding axial length quite large.

\section{Long-Range Interaction}

Almost hundred years ago a discussion started on the concept of local realism, long-range interaction, entangled states, hidden variables and Bell's inequality, in relation to the theories of relativity and quantum mechanics. It included the long-distance interaction between two particle systems and the influence on their intrinsic properties, such as the spin. The associated problems will here be reconsidered in terms of the modes defined in Section 2.

\subsection{Advantageous Points}

Two spatially limited particle models based on the EMS mode can in principle become linked at a long mutual distance by an $S$ mode which is not relativistically limited in its speed of transmission:

- The EMS mode remains relativistic also when the $S$ mode satisfies the quantum mechanical conditions of superluminal long-range interaction.

- The EMS mode possesses a nontransverse (longitudinal) electric field component which could make possible a matching to the $\mathrm{S}$ mode.

- All field strengths $\mathbf{E}$ and the magnetic one $\mathbf{B}$ of the EMS mode are linear functions of a common generating function and its derivatives [1]. A change of sign of the amplitude of the same function therefore changes the sign of all field components, and from symmetry arguments also that of the spin. This would become consistent with the behaviour of entangled states during long-range interaction.

\subsection{Points Requiring Clarification}

There are also points which require further clarification to justify a formally rigorous model:

- The geometrical matching between the spatially limited particle geometries with a long-range linking mechanism of large transverse extension is a 
difficult formal task. This also concerns earlier discussions by other scientists.

- The existence of a flat $S$ mode configuration may also be questioned, in particular concerning its large transverse spatial extension as compared to the cross-sections of the EMS particle models.

\section{Conclusion}

A recently developed revised quantum electrodynamic theory has added some new ideas to the commonly discussed problems of long-range interaction and entangled states. These ideas indicate that the theories of relativity and quantum mechanics do not necessarily have to get into conflict with each other. The same ideas are consistent with causality, and are not related to events taking place backwards in time. But more investigations are required for a rigorous and detailed analysis to be completed. The present investigation has only to be taken as a proposal on this matter.

\section{Conflicts of Interest}

The author declares no conflicts of interest regarding the publication of this paper.

\section{References}

[1] Lehnert, B. (2014) Revised Quantum Electrodynamics. In: Dvoeglazov, V.V., Ed., Contemporary Fundamental Physics, Chs 5, 6, 9, 11.1, 11.2, Nova Science Publishers, New York.

[2] Lehnert, B. (2018) Impacts of Revised Quantum Electrodynamics on Fundamental Physics. Journal of Electromagnetic Analysis and Applications, 10, 106-118. https://doi.org/10.4236/jemaa.2018.105008 